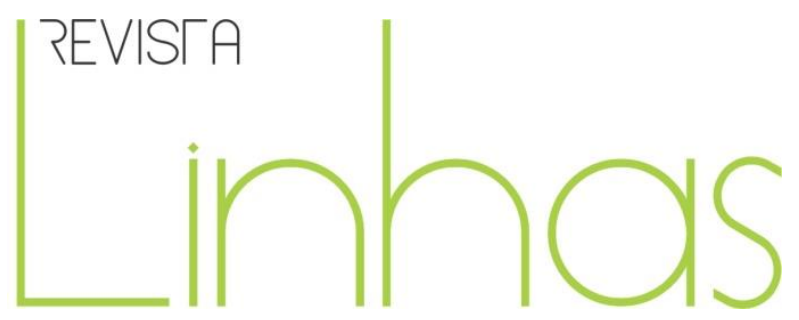

\title{
Metodologías feministas con tecnologías para la gestión de la información en la enseñanza universitaria
}

\section{Resumen}

En nuestras universidades, para avanzar hacia un modelo de enseñanza-aprendizaje que sitúe el alumnado en el centro, es necesario facilitar su aprendizaje autónomo, en colaboración y generando capacidades para la elaboración de proyectos de investigación en perspectiva de género. En este sentido, el proyecto FAAMEF (Facilitando el aprendizaje autónomo sobre metodologías de investigación feminista) se propuso facilitar el aprendizaje autónomo y colaborativo de las metodologías de investigación feminista con la construcción, uso y difusión de un repositorio on-line en Zotero sobre metodologías de investigación feminista. En este artículo presentamos brevemente el proyecto y sus especificidades y nos adentramos en el análisis de la implementación de sus dos primeras fases. Con ello exponemos que los y las alumnas, aunque no sin dificultades y puntos de partida diversos, no sólo han aprendido a seleccionar, organizar y evaluar referencias bibliográficas de forma autónoma y en colaboración satisfactoriamente, sino que han comprendido la utilidad de herramientas como Zotero. Además, han desarrollado la capacidad de pensar críticamente alrededor de los contenidos de las metodologías de investigación feminista.

Palabras clave: Aprendizaje autónomo. Zotero. Metodologías feministas. Innovación docente.

\author{
Núria Vergés Bosch \\ Universitat de Barcelona - \\ Espanha \\ nuria.verges@ub.edu \\ Barbara Biglia \\ Universitat Rovira i Virgili - \\ Espanha \\ barbara.biglia@urv.cat \\ Elisabet Almeda Samaranch \\ Universitat de Barcelona - \\ Espanha \\ elisabet.almeda@ub.edu.fr
}

\section{Para citar este artigo:}

VERGÉS BOSCH, Núria; BIGLIA, Barbara; ALMEDA SAMARANCH, Elisabet. Metodologías feministas con tecnologías para la gestión de la información en la enseñanza universitaria. Revista Linhas. Florianópolis, v. 21, n. 45, p. 165-182, jan./abr. 2020. 


\section{Feminist methodologies with reference technologies in management education}

\begin{abstract}
In our universities, in order to move towards a teaching-learning model that places students in the center, it becomes necessary to facilitate their autonomous learning, in collaboration and by generating capacities for carrying out research with gender perspective. In this sense, the FAAMEF project (Facilitating the autonomous learning of feminist methodologies) set out to facilitate the autonomous and collaborative learning of feminist research methodologies with the construction, use and dissemination of an online repository in Zotero on feminist research methodologies. In this article, we briefly introduce the innovation-teaching project and its specifics, and we delve into the analysis of the implementation of its first two phases. With this, we expose that the students, although not without difficulties and a great diversity of levels of departure, learnt to better select, organize and evaluate bibliographical references in an autonomous and collaborative way. Moreover, they understood the usefulness of tools such as Zotero even if some never used them before. In addition, they developed the ability to think critically about the contents of research methodologies from a gender perspective.
\end{abstract}

Keywords: Autonomous learning. Zotero. Feminist methodologies. Teaching innovation.

\section{Metodologias feministas com tecnologias para gerenciamento de informação na educação universitária}

\begin{abstract}
Resumo
Em nossas universidades, para avançar em direção a um modelo de ensino-aprendizagem que coloque os alunos no centro, é necessário facilitar o aprendizado autônomo, em colaboração e gerando capacidades para a elaboração de projetos de pesquisa na perspectiva de gênero. Neste sentido, o projeto FAAMEF (Facilitando o aprendizado autônomo sobre metodologias de pesquisa feminista) teve o propósito de facilitar o aprendizado autônomo e colaborativo das metodologias de pesquisa feminista com a construção, uso e difusão de um repositório on-line em Zotero sobre metodologias de pesquisa feminista. Neste artigo, apresentamos brevemente o projeto e suas especificidades e entramos na análise da implantação de suas duas primeiras fases. Com isso, expomos que os alunos, embora não sem dificuldades e a partir de pontos de partida diversos, aprenderam não apenas a selecionar, organizar e avaliar referências bibliográficas de forma autônoma e em colaboração de forma satisfatória, mas também entenderam a utilidade de ferramentas como Zotero. Além disso, eles desenvolveram a capacidade de pensar criticamente sobre o conteúdo das metodologias feministas de pesquisa.
\end{abstract}

Palavras-chave: Aprendizagem autônoma. Zotero. Metodologias feministas. Inovação docente. 
Este artículo presenta resultados del proyecto de innovación docente de la Universidad de Barcelona FAAMEF (2014PID-UB/059). Este proyecto está adscrito al grupo de innovación docente consolidado CEFOCID-COPOLIS (GIDCUB-13/140) del Departamento de Sociología de la UB y también se enmarca en la red "Género, Ciudadanía y políticas" (GENCPOLIS- FEM201571218-REDT). También se ha elaborado en el contexto de la INCASI Network, un proyecto europeo Horizon2020(Marie Skłodowska-Curie GA No 691004) coordinado por el Dr. Pedro López. Esta comunicación solo refleja la opinión de las autorías y la agencia no se responsabiliza de su uso ni de la información que contiene.

\section{Introducción}

Aún, y en gran medida, la labor que se desarrolla en nuestras universidades se basa en la memorización del contenido transmitido por el profesorado y en la aplicación práctica únicamente en el aula. Aunque el alumnado suele desarrollar tareas fuera del aula, ello no siempre facilita suficientemente su aprendizaje autónomo, ni su capacidad para la elaboración rigurosa y creativa de proyectos de investigación con perspectiva de género. La investigación feminista parte de las críticas y propuestas epistemológicas feministas. Con ello, comúnmente, se desarrolla en procesos que se sitúan en un dentro-fuera de la academia y con una voluntad transformadora que hace necesarios que los recursos de información se compartan y se mantengan accesibles para la sociedad. Con el desarrollo de la sociedad de la información se han desarrollado plataformas digitales libres que permiten la gestión y difusión de información académica on-line como Zotero y que, además, han sido desarrolladas por comunidades que dejan el conocimiento abierto.

Por todo ello, el proyecto FAAMEF (Facilitando el aprendizaje autónomo sobre metodologías de investigación feminista) tenía como principal objetivo facilitar que el alumnado desarrollara la capacidad de trabajar de forma autónoma a la hora de realizar investigaciones feministas y que, para ello, construyera, utilizara y difundiera nuevos recursos abiertos on-line dirigidos a toda la comunidad universitaria y social mediante el uso de las plataformas tecnológicas.

En una primera fase, se diseñaron y establecieron las estrategias del proyecto por parte del equipo coordinador interuniversitario, interdisciplinar e intergrupal. Una segunda fase se desarrolló en las asignaturas de metodologías de investigación feminista, on-line y presencial, 
del Máster de mujeres, género y ciudadanía del IIEDG (Instituto Interuniversitario de Género de Catalunya) que se desarrollan en las instalaciones de la UB (Universidad de Barcelona). En ello colaboraron SIMReF (Seminario Interdisciplinar de Metodologías de Investigación Feminista) y CEFOCID-COPOLIS (Centro de formación continua e innovación docente del grupo Copolis). En una tercera fase, buscamos su aplicación y uso en varias asignaturas que ya incidían en cuestiones de género en los estudios de Sociología de la UB (Universidad de Barcelona). Finalmente, en una última fase, trabajamos su difusión entre la comunidad académica de otras universidades y la sociedad, especialmente entidades de mujeres y feministas.

En este artículo, después de señalar la importancia de facilitar el aprendizaje autónomo con las TIC de las metodologías de investigación feminista y describir los rasgos principales del proyecto FAAMEF, pretendemos exponer los resultados de la primera y segunda fase del proyecto derivados de la construcción de un grupo y librería Zotero de metodologías de investigación feminista. Con ello las y los alumnos han aprendido a seleccionar, organizar, evaluar y sistematizar la información relacionada con las metodologías de investigación feminista de una manera autónoma, en colaboración y on-line. A la vez, se familiarizaron con el funcionamiento de una plataforma tecnológica como Zotero que permite la organización y la exposición de recursos académicos de forma abierta. Además, se han concienciado sobre la importancia de organizar la información para que luego pueda ser útil para sus investigaciones y de servicio para la comunidad académica y social. Finalmente, han puesto en práctica su capacidad de evaluación y crítica y, con ello, han desarrollado habilidades de pensamiento crítico y ético comprometido con la investigación feminista.

\section{La necesidad de aprender metodologías de investigación feminista de forma autónoma con las tic}

La investigación universitaria adolece de formas de hacer tradicionales que han sido ampliamente cuestionadas por las epistemólogas y metodólogas feministas (ANDERSON, 2015; MARTINEZ et al., 2014). En este sentido, el conjunto de epistemologías feministas, por un lado, critican que la investigación tradicional ha excluido a las mujeres de la investigación, negado su autoridad epistémica, invisibilizado sus voces, así como sus temas de interés y afectación (ANDERSON, 2015; BARTRA, 1998; HARDING, 2004; MARTIN \& MUÑOZ, 2014). Con ello, cuestionan la objetividad, racionalidad y neutralidad que se presupone al quehacer científico y exponen que, históricamente, se han producido teorías que invisibilizan a las mujeres y las 
relaciones de poder entre géneros y producen conocimiento sesgado, androcéntrico, que refuerza jerarquías y no resulta útil para las colectividades oprimidas (HARDING, 2004; BIGLIA \&. VERGÉS, 2016). A su vez, proponen incluir la perspectiva de género en nuestras investigaciones para resolver viejas y nuevas cuestiones, teorías y métodos. Ello implica, además, reflexionar y mostrar las subjetividades, parcialidades y lo situado del conocimiento que se produce (HARAWAY, 1988; MENDIA AZKUE et al., 2014). Finalmente, las propuestas de las epistemologías feministas implican promover investigación transparente, responsable y comprometida para la transformación, también en pro de la igualdad y liberación de género dentro y fuera de la academia (BIGLIA \&. VERGÉS, 2016; MARTINEZ et al., 2014). Entre otras especificidades, la investigación feminista tiende a trabajar mano a mano con el movimiento feminista y a partir y en pro de la ética y agenda feminista (ACKERLY \& TRUE, 2010; MENDIA AZKUE et al., 2014). Por ello, se desarrolla en procesos que se sitúan en un dentro-fuera de la academia que debe dejar abiertos los canales de comunicación entre la academia y sociedad. En este sentido, hace falta que los contenidos y recursos de información que se generan sean accesibles fuera de la academia, además de ser compartidos con el resto de la sociedad con voluntad transformadora (BIGLIA, 2007; VERGÉS et al., 2014; PARDO, 2014).

Como ocurre con la investigación, gran parte de la actividad docente de nuestras universidades sigue desarrollándose de la forma tradicional, en el aula y en el marco de un modelo de transmisión del conocimiento jerarquizado, vertical, fijo y cerrado. Sin embargo, cada vez más, universidades y docentes apostamos por otros modelos más abiertos, horizontales, dinámicos y menos jerárquicos donde las y los estudiantes devienen responsables de su aprendizaje (BLANCHARD \& MUZÁS, 2014). Ello, nos lleva a explorar los mecanismos para facilitar el aprendizaje autónomo del alumnado, un tipo de aprendizaje que suele ser el más ignorado por el profesorado y la innovación docente (HERNANDEZ \& MIÑO, 2012). Según ellos, lo que a opinión de los estudiantes caracteriza el proceso de aprendizaje autónomo sería la búsqueda de información, dotarla de sentido y apropiarse de los conocimientos, aprender más allá de la universidad y de los propios errores, así como posicionarse con criterio propio ante los contenidos. El aprendizaje autónomo, pues, implica la capacidad de aprender por una o uno mismo, tomar el control, autonomía y autorealización que se relacionan con el desarrollo personal (ESCRIBANO, 1995). La capacidad de aprendizaje autónomo, además, es esencial para poder desarrollar proyectos de investigación y, en este caso que nos ocupa, proyectos de investigación feminista. 
En relación a ello, justamente el uso de las nuevas tecnologías de la información y comunicación (TIC) parece que está favoreciendo en mayor medida el aprendizaje autónomo, así como la difusión de contenidos y compartir el conocimiento generado (RODRIGUEZ, 2014). Además, también las mujeres encuentran ventajas en la formación a través y/o con la utilización de las TIC, pues, entre otras cuestiones, implica un acceso y ahorro en términos de tiempo, recursos y desplazamientos y una gestión más flexible del horario de estudio y trabajo (ANDRUSYSZYN \& CRAGG, 2006; BIGLIA Y JIMÉNEZ, 2012). Incluso, más allá de ello, cabe considerar una apuesta de pedagogía ciberfeminista que busque desarrollar un proceso de enseñanza/aprendizaje comprometido con fomentar el intercambio de conocimientos de manera horizontal e independiente de las barreras geográficas, con la autoreflexión y la experiencia previa, con el cuestionamiento de las dinámicas de poder existentes, así como con la comunidad y la política feminista situada que fomente la difracción de conocimientos, experiencias y aprendizajes (LUXAN \& BIGLIA, 2011).

Para todo ello la herramienta para recolectar, organizar, citar y compartir referencias bibliográficas Zotero presenta una serie de ventajas que la convierten en ideal para facilitar el trabajo autónomo y colaborativo sobre metodologías de investigación feminista. Tal y como apuntan otras autorías (AVELLO et al., 2013; BEATTY, 2016; WINDSLOW et al., 2016), herramientas 2.0 como Zotero permiten el trabajo autónomo y colaborativo en red y generan nuevas posibilidades para la educación semipresencial y virtual, así como facilitan proyectos de trabajo en grupo y la participación activa y colaboración entre profesorado, alumnado y entre alumnado. Además, Zotero es una herramienta fácil de usar, de mínimo coste y desarrollada en la comunidad de software libre que se presenta como una aliada útil en el proceso de investigación de docentes y estudiantes de forma integrada al navegador de internet, además de como aplicación específica. Entre sus principales ventajas destacan la facilidad y rapidez en la detección, recolección y gestión de referencias on-line; la posibilidad de trabajar en grupo y compartir los repositorios creados, así como añadir nuevas referencias desde nuestras propias/otras colecciones; resulta muy relevante de cara a fomentar la autoreflexión e incorpora la posibilidad de tomar notas y de etiquetar las referencias con palabras clave; finalmente, prevé la posibilidad de citar (pudiendo elegir entre diferentes estilos) a la hora de elaborar textos, minimizando así las dificultades y errores en cuanto a citación que comúnmente ocurren en nuestras aulas. 
Por ello, como veremos a continuación, donde se presentan los objetivos y estrategias metodológicas del proyecto, decidimos trabajar en torno a la construcción de un repositorio on-line Zotero de metodologías de investigación feminista.

\section{Objetivos y estrategias metodológicas}

Nuestro proyecto de innovación docente buscaba potenciar que el alumnado aprendiera de forma autónoma y colaborativa a través de la construcción, uso y difusión de un repositorio de información on-line en Zotero relacionado con las metodologías de investigación feminista. Por ello, primeramente, era necesario que el alumnado hiciera una búsqueda de una serie de recursos y datos existentes sobre éstas metodologías. Luego, que las y los alumnos hicieran un uso determinado de ello para consolidar el aprendizaje del contenido de las asignaturas implicadas y para potenciar competencias como la selección, organización de la información, el espíritu crítico y la capacidad de analizar casos. Finalmente, y como última tarea, el proyecto incluyó que el alumnado organizara y llevara a cabo la difusión del repositorio on-line Zotero.

En definitiva, también se buscaba promover que las y los alumnos desarrollaran las competencias relacionadas con el tratamiento de la información, es decir, en los procesos de investigación, de selección, de organización y de ordenación, de sistematización, de presentación, de transmisión y de análisis crítico de la misma. Así como y, sobre todo, gracias a la difusión de la base de recursos y datos potenciáramos una transferencia del contenido trabajado en el aula universitaria en la comunidad científica y social para quién pudiera ser de interés y utilidad el trabajo realizado. Así pues, con el proyecto estábamos potenciando que el alumnado se implicara en un aprendizaje que significaría, también, un servicio para la comunidad.

Este proyecto se desarrolló a iniciativa del grupo consolidado de innovación docente CEFOCID-COPOLIS en colaboración con el SIMReF en el que participaron profesoras de distintas universidades del ámbito catalán y vasco que ya participaban en estos grupos previamente. Con ello evidenciamos la elevada transdisciplinariedad, que también caracteriza las metodologías de investigación feminista, y el carácter interuniversitario e intergrupal del proyecto, que facilitaron un mayor uso de los contenidos y su difusión.

El proyecto tuvo una duración de 3 años, 2015-2017, que se distribuyó en 4 fases de implementación. En este artículo nos centraremos en la primera y la segunda fase, que se 
desarrollaron en los cursos 2014-2015 y 2015-2016, y sólo presentaremos resultados al respecto de estas dos primeras fases, para poder profundizar en ello.

En una primera fase se diseñó el proyecto, las guías y materiales de acompañamiento para el alumnado, así como las principales estrategias a seguir para alcanzar los objetivos formativos y operativos del proyecto. En esta fase se implicó a 3 professoras ya implicadas en SIMrEF de distintas universidades catalanas y vascas. Profesorado de las asignaturas de "Metodologías de investigación feminista" del máster de género interuniversitario del IIEDG, aunque adscrito a la Universidad de Barcelona, en su modalidad on-line y presencial. También al profesorado de "Metodologías I" de la Universidad del País Vasco (UPV/EHU) "Fundamentos de la metodología de investigación feminista" y "Miradas y aplicaciones feministas en la metodología cualitativa" de la Universidad Rovira y Virgili (URV). El profesorado implicado provenía pues de SIMReF y, a su vez, de la universidad de Barcelona (UB), de la Universidad Rovira y Virigili (URV) y de la Universidad del País Vasco (UPV/EHU) y se constituyó como kernel coordinador del proyecto en su conjunto.

Respecto a las tareas relacionadas con la primera fase, de coordinación y diseño de las actividades, se generaron una serie de presentaciones en encuentros de SIMReF y de CEFOCIDCOPOLIS tanto sobre el estado y avance del proyecto FAAMEF como para discutir los contenidos de las guías y recursos a utilizar en la fase 2, 3 y 4. A través de diversos encuentros sucesivos de SIMReF Catalunya donde específicamente se trabajó en algún aspecto del proyecto-que fueron 3 durante el curso 2015-2016, además de 1 encuentro general de SIMReF en Hernani con SIMREF Catalunya-Euskadi- se discutieron diferentes herramientas posibles, así como sus tiempos y distribución de tareas del proyecto en general. Por las diversas ventajas ya anotadas, así como por el hecho de constituirse como un recurso en software libre se apostó por la herramienta Zotero (AVELLO et al., 2013). También se definió y diseño la primera guía de uso de Zotero para el alumnado, así como los primeros pasos para las fichas para la evaluación de materiales y artículos de metodología de investigación feminista. Sobre esta base, se hicieron varias revisiones on-line sobre las diversas posibilidades de etiquetaje de las referencias, estableciendo diferentes posibilidades de familias y palabras clave asociadas para facilitar el trabajo de las alumnas en la fase 2 y 3 . Ello nos llevó a un work in progress que se dejó abierto a las intervenciones del alumnado que acabaría conformando la herramienta y los contenidos finales del repositorio on-line. Finalmente, aprovechando la formación en metodologías feministas para el profesorado de la Universidad de Barcelona (UB) que impartimos dos de las autoras, se plasmaron sus contenidos en un artículo académico que, más 
adelante, sirvió para las dos últimas fases del proyecto. Así, se convirtió en un artículo introductorio para el fomento del uso de la librería Zotero sobre metodologías feministas, y de la perspectiva de género en la investigación en general (BIGLIA \& VERGÉS, 2016).

Una segunda fase consistió en la selección, organización y evaluación de materiales, como son vídeos, artículos, documentales, libros etc. para el aprendizaje y uso de las metodologías de investigación feminista por parte del alumnado. En esta fase se implicaron las y los alumnos de las asignaturas optativas de metodología de investigación feminista del máster de mujeres, género y ciudadanía del IIEDG para el curso 2015-2016, de primer semestre, tanto de la modalidad presencial como de la modalidad on-line. Además, apoyó en la realización del proyecto, una alumna de último año del grado Sociología de la UB como parte de su beca de colaboración con CEFOCID-COPOLIS. En este máster interuniversitario cuyo "objetivo general [...] es dar una formación en los estudios sobre mujeres y género que sea científicamente rigurosa y al mismo tiempo aplicable a problemáticas de interés social", participan ocho universidades catalanas: Universitat de Barcelona (coordinadora), Universitat Autònoma de Barcelona, Universitat de Girona, Universitat Politècnica de Catalunya, Universitat Rovira i Virgili y Universitat de Vic, Universidad de Lleida y Universitat Pompeu Fabra. El estudiantado proviene de diferentes disciplinas, principalmente de ciencias sociales y humanísticas, y la mayoría han cursado asignaturas metodológicas durante el grado. A diferencia de los estudios de grado que en cierta medida se cursan en catalán, el máster se lleva a cabo en lengua española ya que las aulas cuentan con alumnado de una gran diversidad de orígenes (sobre todo de Latinoamérica y del Estado español). Destacar por otra parte que asisten a este máster también personas con una larga trayectoria profesional y/o activista con lo cual la media de edad suele ser superior a la media de otros másteres. Finalmente, si bien estos estudios están abiertos a personas de cualquier género, los cishombres suelen ser muchos menos. Específicamente para nuestras asignaturas de metodologías feministas del curso 2015-2016, en que realizamos estas fases del proyecto, 17 personas se matricularon en la asignatura de metodología en la modalidad presencial y 9 en la versión on-line, de todas ellas solo el $11 \%$ eran hombres. Se escogieron estas asignaturas por ser las que directamente trabajan los contenidos de las metodologías de investigación feminista y, así, poder actuar directamente en su alumnado. Mediante este primer año, y desarrollando esta tarea necesaria para la evaluación continuada, las y los alumnos tuvieron que aprender a seleccionar, organizar y sistematizar la información de una manera autónoma. A la vez, se familiarizaron con el funcionamiento de una plataforma tecnológica que permite la organización y la exposición de 
recursos abiertos académicos como Zotero. Además, se buscó que se concienciaran de la importancia de organizar la información para que luego pudiera ser de servicio para la comunidad académica y social.

En la segunda fase, por un lado, en el marco de la asignatura de metodologías de investigación feminista presencial se llevó a cabo una primera evaluación de materiales de investigación feminista. Para ello, siguiendo las explicaciones de la profesora y a través de unas fichas guía que se habían elaborado previamente las alumnas deberían recopilar, seleccionar, organizar y evaluar una serie de materiales bibliográficos y los en unas fichas en formato Excel que, posteriormente, serían volcadas a la herramienta Zotero y los comentarios incluidos como notas. Estas fichas contenían los siguientes campos:

-Nombre y apellidos de la estudiante

-Referencia completa en formato APA

-Palabras clave metodológicas (Hasta 4)

-Link al texto completo

-Resumen de las principales apuestas metodológicas del material

-Elementos epistemológicos poco claros

-¿Hasta qué punto el texto ahonda en propuestas feministas?

-Otros comentarios a añadir

El trabajo con las fichas no sólo permitía a las alumnas aprender de forma autónoma a seleccionar, organizar, etiquetar y evaluar materiales bibliográficos, sino que ya las familiarizaba con los formatos de citación académicos y, sobre todo, provocaba la reflexión crítica en torno a las metodologías de investigación feministas y sus especificidades.

Por otro lado, en el marco de la asignatura de metodologías de investigación feminista on-line y con el apoyo de la estudiante de grado de sociología colaboradora docente, el trabajo se centró no sólo en la selección, organización y evaluación de materiales, sino, sobre todo, la inclusión de referencias de metodología feminista en el grupo Zotero, es decir, en la construcción del repositorio on-line Zotero ${ }^{1} \mathrm{y}$, por ello, en el aprendizaje de uso de herramientas TIC de gestión y colaboración bibliográfica muy útiles para la realización de proyectos de investigación. Además, se volcaron los materiales y evaluaciones de la asignatura presencial, así como se complementó con la bibliografía recomendada del curso y el repositorio

\footnotetext{
${ }^{1}$ Puede consultarse la librería en el siguiente link:

https://www.zotero.org/groups/414156/metodologiasfeministas/items
} 
audiovisual SIMReF. Todo ello resultó en un repositorio on-line sobre metodologías de investigación feminista construido de forma colaborativa de más de 100 entradas.

Finalmente, en el marco de la asignatura y para fomentar el intercambio de experiencias y el aprendizaje en colectivo se creó un foro on-line para que el alumnado pudiera compartir y evaluar sus experiencias en relación a la utilidad de Zotero para la gestión de información y sus ventajas e inconvenientes, así como el grado de satisfacción y aprendizaje percibidos con la experiencia.

\section{Resultados: la experiencia de selección, organización y evaluación de materiales para la construccion de una librer}

Teniendo en cuenta todo lo anterior, en este apartado nos centramos en mostrar los resultados la experiencia de construcción de un repositorio on-line con Zotero sobre metodologías de investigación feminista desarrollado en el marco del proyecto de innovación docente. En este sentido, mostramos las actividades, y sus resultados e impactos, que se han desarrollado en las asignaturas de metodologías de investigación feminista, presencial y online, del máster de mujeres, género y ciudadanía del IIEDG. El proceso de selección, gestión y evaluación bibliográfica, así como de construcción de la herramienta Zotero y su uso ha evidenciado una serie de resultados adicionales, así como nos permite evaluar la experiencia de innovación docente realizada para ambas asignaturas.

En primer lugar, cabe destacar que al analizar las evaluaciones de las referencias seleccionadas se visibiliza el aprendizaje adquirido en cuanto a los contenidos de las asignaturas, pero también en las competencias de desarrollo de pensamiento crítico y compromiso ético en relación a la investigación y, específicamente, la investigación feminista. El aprendizaje se va adquiriendo a lo largo de todo el proceso. Sin embargo, mejora aún más con la realización de tareas específicas como el análisis con perspectiva feminista de los textos elegidos a analizar como mostramos a continuación:

Esta autora, en este libro, ofrece un marco teórico y metodológico para analizar el género, la construcción identitaria y la capacidad de agencia de las personas para modificar el entorno y su propia trayectoria. Según la autora, todos los procesos (sociales, personales y mentales) se expresan mediante el cuerpo. Además, destaca el reconocimiento de la capacidad de agencia de todas las personas, es decir, la capacidad de transformar la realidad que nos rodea. Esta visión conlleva una manera de análisis de las personas en positivo, nunca como víctimas. (C.M.D) 
Me parece una propuesta metodológica eminentemente feminista, no solo porque se enuncia desde ahí, ya que la posibilidad de nombrarnos y autoconstruirnos es un rasgo propio del feminismo, sino que además, apuesta la construcción de un conocimiento generado a partir de una red de relaciones sociales donde está incluida la persona que investiga, rompiendo con la propuesta de la ciencia tradicionalmente androcéntrica que sostiene el conocimiento ha de ser neutral, generalizable y desligado del contexto. Esta crítica a los valores implícitos tanto en contenido pero también en los procedimientos mismos de investigación, es inminentemente feminista, así como ligar la experiencia y acción, volviendo ésta última, fundamental en el proceso, así como el proceso mismo. Por otro lado el intento de la mirada interseccional complejiza y localiza el análisis feminista, ya que rompe con la mirada occidental y androcéntrica de la "otra", en este caso, interseccionadas en experiencias y conocimientos. (A.G.A)

En segundo lugar, cabe constatar que si bien se trata de un máster aún hay alumnas que no estaban suficientemente familiarizadas con herramientas de gestión bibliográfica como Zotero o, incluso, nunca habían tenido conocimiento de ellas o la oportunidad de usarlas. Ello pues, es una muestra de la necesidad de seguir fomentando el uso de herramientas TIC para la selección, gestión y uso de materiales bibliográficos en nuestras universidades y tareas docentes y se ve reflejada en la siguiente cita:

He de empezar por comentar que no tenía experiencia previa con herramientas de este tipo. He visto que otros compañeros/as hablan con soltura de Zotero y de Mendeley pero para mí es una auténtica novedad, sabía que existían pero no había trabajado nunca con ellas. (C.M.D)

Por ello, en tercer lugar, anotamos que sigue siendo necesario elaborar materiales guía, tanto las informaciones generales encontradas en la web de Zotero como las guías elaboradas para este proyecto han resultado de ayuda para desarrollar la actividad por parte de los y las alumnas. Ello implica que, para lograr un aprendizaje autónomo que pueda incluso desarrollarse fuera de la universidad y en colectivo, es necesario acompañar el aprendizaje.

En cuanto a las etiquetas, tal y como ya han apuntado las compañeras, es recomendable seguir la guía de etiquetado propuesta. Tal y como sucede en cualquier ejercicio de categorización y sistematización de la información (como sería el caso, en otra fase de investigación, de la codificación de textos), es muy útil disponer de un listado de referencia que permita homogeneizar las etiquetas $y$, en consecuencia, poder consultar de manera más satisfactoria. Especialmente si se tiene en cuenta que en un grupo de Zotero son varias las personas que pueden añadir y editar entradas y que, por tanto, el etiquetado se regirá por tantos criterios diferentes como investigadoras participen. (M.G.G) 
Persisten algunas dificultades con algunas aplicaciones e, incluso, con el idioma de la mayoría de ayudas relacionadas con Zotero. En este acompañamiento, cabe pues la elaboración de guías más accesibles y más amigables. Algunas dificultades se relacionan, por un lado, con el funcionamiento mismo de la herramienta, en cuanto a sincronización, conversión de formato de documentos etc... Por otro lado, al hecho que la mayoría de explicaciones de la propia herramienta se hacían en inglés. Finalmente, a las dificultades de análisis de información, como, por ejemplo, reflexionar y decidir unas etiquetas y/o clasificación determinada.

Si bien es cierto que hemos contado con una "Guía de instalación e introducción de materiales en Zotero" muy detallada y útil, en mi caso he de decir que, aun así, me ha sido muy dificulto manejarme con el programa. $Y$, creo que dichas dificultades e inconvenientes pueden estar relacionadas con el idioma establecido (Inglés). Aún así, una vez conseguido acceder al grupo Zotero de la asignatura, considero que los pasos que hemos de seguir para introducir la bibliografía (siguiendo los iconos e instrucciones) son poco claros. De hecho, no he conseguido comprender como introducir una bibliografía en pdf ni desde una página web (aun siguiendo con las instrucciones de la guía y las soluciones que la misma página ofrece). Y, del mismo modo que comenta el compañero, me ha sido imposible colgar ninguno de los documentos que tenía en mi biblioteca (los he tenido que colgar manejándome dentro del mismo grupo creado por la asignatura). (C.P.M)

Yo me he visto con una dificultad (por el tema de la sincronización) y lo tenía que hacer manualmente, pero supongo que es aún más fácil de usar si tienes el DOI ... Pero, en este punto, sí he decir que he tenido problemas (se complica una herramienta que te ha de hacer el trabajo más fácil). Pero han sido problemas que seguro tienen que ver con el ordenador (o sea, técnicos y no del programa mismo). También he tenido dificultades, ahora sí hablando del programa en sí, para llenar algunos campos en blanco a la hora de registrar una referencia. Había campos que he dejado completamente en blanco porque no sabía muy bien dónde encontrar esa información. Por ejemplo, cuando registraba un artículo académico, dejaba en blanco los apartados Accessed, archive, loc.in archive ... No sé si esto está bien o mal. Etiquetar las referencias no me ha resultado complicado, pero sí es verdad que muchas veces me encontraba en la problemática de no saber muy bien qué etiquetas eran claves y válidas para cada artículo. Hay artículos que, al leer el resumen (abstract) piensas que va de un tema y luego aquel tema que tú pensabas que era primordial sólo ocupa un pequeño espacio en todo el artículo. (I.N.A)

Aunque algunas de los y las alumnas estuvieran familiarizadas con herramientas de gestión bibliográfica no lo estaban tanto con herramientas como Zotero. Al ser herramientas libres tienen ventajas añadidas para su acceso, para compartir el conocimiento generado y, 
sobre todo, de cara al aprendizaje autónomo a la vez que colaborativo. Ello también se visibilizó, interiorizado y apuntado por el alumnado, como muestra la cita siguiente:

Considero que los programas de almacenaje de bibliografía son una herramienta fundamental en la investigación. Hace años en la Universidad Rovira i Virgili, donde estudié y actualmente trabajo, puso accesible para sus usuarias el programa de Refworks. Este programa era una base de datos donde poder exportar e importar las referencias de aquellos trabajos que se consultaban. De esta manera era más sencillo poder volver a la documentación consultada. Hace ya dos años la URV observó que este programa estaba un tanto obsoleto por lo que optó por migrar hacia Mendeley, otro programa que esta vez permitía incorporar los artículos a la nube digital. El gestor bibliográfico Zotero nos da la posibilidad de acceder a bibliografía consultada y colgada por otras investigadoras. La diferencia con Mendeley, por ejemplo, es su capacidad de establecer grupos de trabajo como el nuestro. Este es, sin duda, la característica fundamental y más importante que destacaría del programa Zotero, es decir, el trabajo en equipo. Esto nos permite acceder a artículos inhóspitos que por otros medios sería complejo acceder y por consecuencia establecer grupos de trabajo. Por otro lado, también es interesante como el gestor bibliográfico Zotero también permite introducir directamente las referencias en programas de ofimática como Word. De la misma manera nos permite acceder e incorporar referencias desde muchos portales digitales. Dar continuidad a una base bibliográfica en nuestra investigación personal considero que es clave y práctica para la elaboración laboral. (G.P.P)

Cabe decir, como se apuntó anteriormente, que los elementos apuntados de acceso libre, así como las posibilidades de colaboración y de uso autónomo y compartido, se constituyen como elementos clave para la investigación feminista. Además, en cuarto lugar, y como vamos viendo pues, se concienciaron de la importancia de utilizar herramientas TIC para la gestión y uso de referencias bibliográficas para su aprendizaje, sobre todo de cara a la realización de proyectos de investigación en colaboración.

Cuando se lleva a cabo una investigación se consulta una gran variedad de bibliografía, tanto para la justificación teórica como para la presentación de la metodología que se va a llevar a cabo. Es por ello que, la práctica con Zotero considero que es muy beneficiosa porque, al menos en mi caso, me ha permitido separar la información y centrarme en aquella bibliografía que es la base de la metodología que voy a llevar a cabo en mi estudio. (C.P.M)

Valoro el ejercicio de compartir algunas referencias bibliográficas que puedan ser de interés a las compañeras en la plataforma Zotero como una práctica muy positiva hacia el aprendizaje académico colaborativo. A lo largo de una investigación, y en especial en los análisis bibliográficas, se consulta y trabaja con un gran número de documentos que requieren, 
como todo, orden y sistematización. Las herramientas de gestión de referencias como Zotero son casi imprescindibles en la investigación, y la posibilidad de que ésta sea compartida en una plataforma colaborativa como los grupos, esencial para la investigación feminista. (M.G.G)

Para acabar, y como se ha ido viendo, creemos relevante anotar que para la mayoría de alumnas la práctica con Zotero fue satisfactoria. Aunque en algunos casos se expresaron dificultades como vimos, no implicaron una insatisfacción general ni el abandono de las tareas. Al menos, sí que para la totalidad de las alumnas este ejercicio, las guías y la herramienta utilizada fueron consideradas útiles para su aprendizaje y para la realización de futuros trabajos de investigación feminista.

Creo que los resultados son satisfactorios, pero pienso que cuando realmente te das cuenta de la importancia de Zotero es cuando estás trabajando en un artículo propio o en una tesis ... o una investigación individual o colectiva. Bueno, en equipo creo que todavía son más obvias las ventajas, por lo que decía de trabajar en línea (la red). Es, en definitiva, una herramienta para hacer más fácil el trabajo de seleccionar y recopilar información sobre un tema (en este caso, de investigación feminista). Creo que hay que seguir trabajando sobre esta base. (I.N.A)

\section{Conclusiones}

Con el proyecto FAAMEF buscamos mejorar la capacidad del alumnado para realizar proyectos de investigación, y, específicamente de investigación feminista. Buscábamos avanzar en este sentido, a través de la selección, gestión y evaluación de información y la construcción de un repositorio on-line sobre metodologías de investigación feminista. Ello nos sitúa con las universidades y profesorado comprometido con la mejora e innovación docente para avanzar en un aprendizaje centrado en el alumnado. Aunque poco considerado en la innovación docente, el aprendizaje autónomo y colaborativo debe ser facilitado, sobre todo, si buscamos mejorar la capacidad de llevar a cabo proyectos de investigación con perspectiva de género. En este artículo, además de presentar las principales características del proyecto FAAMEF, hemos analizado los resultados de las dos primeras fases. En este sentido, nos hemos centrado en mostrar el proceso y los principales resultados de, por un lado, la coordinación y el diseño de materiales y estrategias guía para el alumnado y, por el otro, las actividades en torno a la selección, gestión y evaluación de referencias y la construcción del respositorio online de metodologías de investigación feminista en Zotero.

Cabe destacar, en primer lugar, que al realizar los ejercicios propuestos de evaluar y etiquetar los materiales seleccionados el alumnado ha desarrollado capacidades de 
pensamiento crítico y perspectiva feminista imprescindibles para la tarea investigadora y, aún más, para la investigación feminista. En segundo lugar, cabe anotar que el trabajo de coordinación y elaboración de materiales guía iniciales por parte del equipo de coordinación del proyecto ha sido fundamental para que las actividades del alumnado fueran más satisfactorias y, sobre todo, para facilitar el aprendizaje autónomo de los y las alumnas de metodologías de investigación feminista, tanto en torno a los contenidos de la asignatura como en el uso de herramientas TIC para la gestión bibliográfica. En tercer lugar, trabajar en la construcción y con herramientas como Zotero ha ayudado a que los y las alumnas comprendieran la utilidad de una buena selección y gestión de referencias bibliográficas con las TIC y para desempeñar investigaciones de rigor, así como valorar las posibilidades del trabajo colaborativo.

Así, pues, aunque algunas barreras siguen presentes como la diferencia de puntos de partida del alumnado, el conocimiento del inglés o las dificultades al ahondar en las posibilidades de las herramientas TIC para construir un repositorio on-line en Zotero, en general ha mejorado el aprendizaje y conocimientos de los y las alumnas alrededor de las metodologías de investigación feminista y sus capacidades para el trabajo autónomo, colaborativo y crítico. Además, teniendo en cuenta que siguen siendo escasos los recursos académicos abiertos y feministas on-line y, así, sus posibilidades de utilización en la academia y la sociedad, el desarrollo de una librería Zotero on-line contribuyó a ello. De hecho, se utilizó en fases posteriores del proyecto y sigue utilizándose en diversas asignaturas y talleres dentro y fuera de la academia hoy en día.

\section{Referencias}


ANDERSON, Elizabeth. Feminist Epistemology and Philosophy of Science. Stanford Encyclopedia of Philosophy. Edición 2015. Recuperado de: http://plato.stanford.edu/entries/feminismepistemology/.

ANDRUSYSZYN, Mary-Anne., \& CRAGG, Betty C. E. Promoting Comfort in E-Learning for Professional Women: Examining Access, Language and Culture, Learning Preferences and Role Integration. International Journal of Instructional Technology and Distance Education, 3(1), 3140, 2006.

AVELLO, Raudell; MARTIN, Ibrahim; DÍAZ, Magaly \& CLAVERO, Maria Isabel. Zotero, más allá de un gestor bibliográfico. Una experiencia con los docentes y nuevas metas. DIM: Didáctica, Innovación y Multimedia, (25), 1-13, 2013.

BARTRA, Eli. Reflexiones metodológicas. In Eli Bartra (comp.), Debates en torno a una metodología feminista, Programa Universitario de Estudios de Género-UNAM, México, 141-158, 1998.

BEATTY, Joshua. F. Zotero: A Tool for Constructionist Learning in Critical Information Literacy. In Pagowsky, Nicole \& McElroy, Kelly (Ed.). Critical Library Pedagogy Handbook. Chicago:

Association of College and Research Libraries, 215-221, 2016.

BIGLIA, Barbara. Desde la investigación-acción hacia la investigación activista feminista. In ROMAY, José (Coord.). Perspectivas y retrospectivas de la Psicologia Social en los albores del siglo XXI. Madrid: Biblioteca Nueva, 415-422, 2007.

BIGLIA, Barbara \& JIMÉNEZ, Edurne. Los desafíos de la pedagogía cyberfeminista: un estudio de caso. Athenea digital: revista de pensamiento e investigación social, 12(3), 71-93, 2012.

BIGLIA, Barbara, \& VERGÉS BOSCH, Núria. Cuestionando la perspectiva de género en la investigación. REIRE. Revista d'Innovació i Recerca en Educació, 9(2), 12-29, 2016.

BLANCHART GIMÉNEZ, Mercedes, \& MUZÁS RUBIO, María Dolores. Claves para un cambio de paradigma en las aulas universitarias. In FOMBONA CADAVIECO, Javier \& CALDEVILLA DOMINGUEZ, David Nuevas formulaciones de los contenidos docentes. Madrid: McGraw Hill Education, 2014, p. 87-100.

ESCRIBANO GONZÁLEZ, Alicia. Aprendizaje cooperativo y autónomo en la enseñanza universitaria. Enseñanza \& Teaching: Revista Interuniversitaria De Didáctica, 13, 89-104, 1995.

HARAWAY, Donna. Situated knowledges: The science question in feminism and the privilege of partial perspective. Feminist studies, 14 (3).,575-599, 1988.

HARDING, Sandra. The feminist standpoint theory reader. Nueva York: Routledge, 2004.

HERNANDEZ, Fernando \& MIÑO, Raquel. Indagar sobre las concepciones de los estudiantes en torno a la experiencia de aprendizaje autónomo. Revista del Congrés Internacional de Docència Universitària i Innovació (CIDUI), 1(1), 2012.

LUXÁN SERRANO, Marta \& BIGLIA, Barbara, Pedagogía cyberfeminísta: Entre utopía y realidades. Teoría de la Educación, 12(2), 18, 149-183, 2011. 
MARTIN PALOMO, Teresa., \& MUÑOZ TERRÓN, José. M. Epistemología, metodología y métodos. ¿Qué herramientas para qué feminismo? Reflexiones a partir del estudio del cuidado. Quaderns de Psicologia, 16(1), 35-44, 2014.

MARTINEZ, Luzma; BIGLIA, Barbara., LUXÁN, Marta., FERNÁNDEZ, Cristina., AZPIAZU, Jokin., \& BONET, Jordi. Experiencias de investigación feminista: propuestas y reflexiones metodológicas. Athenea digital: revista de pensamiento e investigación social, 14 (4), 3-16, 2014.

PRADO, Lena. Repositorio on-line de material audiovisual de metodología de investigación feminista. Athenea digital: revista de pensamiento e investigación social, 14(4), 339-342, 2014.

RODRIGUEZ RUIZ, Juan Roger.Los recursos TIC favorecedores de estrategias de aprendizaje autónomo: el estudiante autónomo y autorregulado. In Crescendo, 5(2), 233-252, 2014.

VERGÉS BOSCH Núria, HACHE, Alex, \& CRUELLS LOPEZ, Eva. Ciberfeminismo de investigacción con y entre tecnoartistas y hackers. Athenea digital: revista de pensamiento e investigación social, 14(4), 153-180, 2014.

WINSLOW, Rachel. R., SKRIPSKY, Sarah \& KELLY, Savannah. L. Not just for citations: Assessing Zotero while reassessing research. In D'ANGELO, Barbara; JAMIESON, Sandra; MAID, Barry \& WALKER Janice R. Information literacy: Research and collaboration across disciplines. Fort Collins: WAC Clearinghouse and University Press of Colorado, 299-316, 2016. 